\title{
Theory of quasi-stationary kinetic dynamos in magnetized accretion discs
}

\section{Claudio Cremaschini ${ }^{1,2}$, John C. Miller ${ }^{1,2,3}$ and Massimo Tessarotto ${ }^{4,5}$}

${ }^{1}$ International School for Advanced Studies, SISSA, Trieste, Italy

${ }^{2}$ INFN, Trieste Section, Trieste, Italy

\author{
${ }^{3}$ Department of Physics (Astrophysics), University of Oxford, Oxford, U.K. \\ ${ }^{4}$ Department of Mathematics and Informatics, University of Trieste, Trieste, Italy \\ ${ }^{5}$ Consortium for Magnetofluid Dynamics, University of Trieste, Trieste, Italy
}

\begin{abstract}
Magnetic fields are a distinctive feature of accretion disc plasmas around compact objects (i.e., black holes and neutron stars) and they play a decisive role in their dynamical evolution. A fundamental theoretical question related with this concerns investigation of the socalled gravitational MHD dynamo effect, responsible for the self-generation of magnetic fields in these systems. Experimental observations and theoretical models, based on fluid MHD descriptions of various types support the conjecture that accretion discs should be characterized by coherent and slowly time-varying magnetic fields with both poloidal and toroidal components. However, the precise origin of these magnetic structures and their interaction with the disc plasmas is currently unclear. The aim of this paper is to address this problem in the context of kinetic theory. The starting point is the investigation of a general class of Vlasov-Maxwell kinetic equilibria for axi-symmetric collisionless magnetized plasmas characterized by temperature anisotropy and mainly toroidal flow velocity. Retaining finite Larmor-radius effects in the calculation of the fluid fields, we show how these configurations are capable of sustaining both toroidal and poloidal current densities. As a result, we suggest the possible existence of a $k i$ netic dynamo effect, which can generate a stationary toroidal magnetic field in the disc even without any net radial accretion flow. The results presented may have important implications for equilibrium solutions and stability analysis of accretion disc dynamics.
\end{abstract}

Keywords. kinetic theory, accretion disks, plasmas, magnetic fields

\section{Introduction: magnetic fields in accretion discs}

In this paper basic issues concerned with the origin and the structure of magnetic fields in accretion disc (AD) plasmas are discussed, with particular reference to the dynamo phenomenon which leads to the self-generation of the magnetic field appearing in these systems. More precisely, we address the problem of the generation of both the poloidal and toroidal components of the AD magnetic field as a consequence of plasma currents produced purely by collisionless and quasi-stationary kinetic mechanisms.

Experimental observations and theoretical models based on fluid MHD descriptions suggest that accretion discs should be characterized by coherent and slowly time-varying magnetic fields with both poloidal and toroidal components (see for example Frank et al., 2002 and Szuszkiewicz \& Miller, 2001). An interesting development within this context has been the work by Coppi (2005) and Coppi \& Rousseau (2006), who showed that stationary magnetic configurations in AD plasmas, for both low and high magnetic energy densities, can exhibit complex magnetic structures characterized locally by plasma rings with closed nested magnetic surfaces. However, even the most sophisticated fluid models are still not able to give a satisfactory explanation for all of the complexity of the phenomena arising in these systems. In particular, the precise origin of these magnetic structures and their interaction with the disc plasmas remains unclear. This is especially true for what concerns the toroidal magnetic field. Usually it is thought that such fields are the result of non-stationary processes associated with some ongoing instabilities in the plasma (possibly the same ones responsible for the accretion), but the 
actual mechanisms for their generation are still a matter of debate. In contrast with these fluid descriptions, the aim of this paper is to address this problem in the context of kinetic theory, which can provide a description of the plasma dynamics at a more fundamental level (Cremaschini et al., 2008 and 2010). The work is based on results presented in a recent paper by Cremaschini et al. , (2010), concerning the investigation of a general class of asymptotic (i.e., quasi-stationary) Vlasov-Maxwell kinetic equilibria for axi-symmetric collisionless magnetized plasmas characterized by temperature anisotropy and mainly toroidal flow velocity. Note that what is meant here by the term "equilibrium" is in general a stationary-flow solution. Retaining finite Larmor-radius effects in the calculation of the fluid fields, we show how these configurations are capable of sustaining both toroidal and poloidal current densities. As a result, for these configurations of the magnetic field, we conjecture the possible existence of a quasi-stationary kinetic dynamo effect which can generate a stationary toroidal magnetic field in the disc even without any net radial accretion flow. The theory presented is of interest for improving our understanding of magnetic fields in accretion discs, concerning both their local structure and generation mechanisms. These results may also have important implications for further studies of the equilibrium solutions and the stability analysis of accretion disc dynamics.

\section{Basic assumptions and notation}

For what concerns the notation adopted and the basic assumptions about the AD plasma and the magnetic field configuration, we refer to the paper by Cremaschini et al. , (2010). The same holds also for the meaning of the dimensionless parameters $\varepsilon, \varepsilon_{M}, \delta$ and $\delta_{T s}$ used in constructing the asymptotic kinetic theory and its relevant expansions. For the sake of clarity, we recall that the present analysis is restricted to the particular situation where the equilibrium magnetic field $\mathbf{B}$ admits, at least locally, a family of nested axi-symmetric closed toroidal magnetic surfaces $\{\psi(\mathbf{r})\} \equiv\{\psi(\mathbf{r})=$ const. $\}$, where $\psi$ denotes the poloidal magnetic flux of B (see Coppi (2005), Coppi \& Rousseau (2006) for a proof of the possible existence of such configurations in the context of astrophysical accretion discs; see also Cremaschini et al. , (2010) for further discussion of this). In this situation, a set of magnetic coordinates $(\psi, \varphi, \vartheta)$ can be defined locally, where $\vartheta$ is a curvilinear angle-like coordinate on the magnetic surfaces $\psi(\mathbf{r})=$ const. In particular, we shall assume that the magnetic field is slowly varying in time and of the form

$$
\mathbf{B} \equiv \nabla \times \mathbf{A}=\mathbf{B}^{\text {self }}\left(\mathbf{r}, \varepsilon_{M} t\right)+\mathbf{B}^{e x t}\left(\mathbf{r}, \varepsilon_{M} t\right),
$$

where $\mathbf{B}^{\text {self }}$ and $\mathbf{B}^{\text {ext }}$ denote the self-generated magnetic field produced by the AD plasma and a non-vanishing external magnetic field produced by the central object. We also take the self field to be the dominant component, while $\mathbf{B}^{\text {self }}$ and $\mathbf{B}^{\text {ext }}$ are defined as

$$
\begin{aligned}
& \mathbf{B}^{\text {self }}=I\left(\mathbf{r}, \varepsilon_{M} t\right) \nabla \varphi+\nabla \psi_{p}\left(\mathbf{r}, \varepsilon_{M} t\right) \times \nabla \varphi, \\
& \mathbf{B}^{e x t}=\nabla \psi_{D}\left(\mathbf{r}, \varepsilon_{M} t\right) \times \nabla \varphi,
\end{aligned}
$$

where $\mathbf{B}_{T} \equiv I\left(\mathbf{r}, \varepsilon_{M} t\right) \nabla \varphi$ and $\mathbf{B}_{P} \equiv \nabla \psi_{p}\left(\mathbf{r}, \varepsilon_{M} t\right) \times \nabla \varphi$ are the toroidal and poloidal components of the self-field.

\section{Asymptotic stationary solution and analytical expansion}

In this section we briefly summarize the solution for the asymptotic stationary kinetic distribution function (KDF) for the case considered here, and its appropriate analytic expansion for describing strongly magnetized collisionless AD plasmas in which the temperature is anisotropic (Cremaschini et al., 2010). An extended treatment can be found in Cremaschini et al. (2010). A convenient solution for the KDF in this configuration is given by

$$
\widehat{f_{* s}}=\frac{\eta_{s}}{\left(2 \pi / M_{s}\right)^{3 / 2}\left(T_{\| * s}\right)^{1 / 2} \widehat{T}_{\perp s}} \exp \left\{-\frac{H_{* s}}{T_{\| * s}}-\frac{m_{s}^{\prime} B^{\prime}}{\widehat{\Delta_{T_{s}}}}\right\}
$$


which we refer to as the Generalized bi-Maxwellian KDF, where $\frac{1}{\widehat{\Delta_{T_{s}}}} \equiv \frac{1}{\widehat{T}_{\perp s}}-\frac{1}{T_{\| * s}}$, with the following kinetic constraints (Cremaschini, 2010): $\frac{\eta_{s}}{\widehat{T}_{\perp s}}=\widehat{\beta_{* s}}\left(\psi_{* s}\right), T_{\| * s}=T_{\| * s}\left(\psi_{* s}\right)$, $\frac{B^{\prime}}{\widehat{\Delta_{s}}}=\widehat{\alpha_{* s}}\left(\psi_{* s}\right)$ and $H_{* s}=E_{s}-\frac{Z_{s} e}{c} \psi_{* s} \Omega_{s}\left(\psi_{* s}\right)$. Here $\psi_{* s}^{\perp s}$ is proportional to the canonical momentum, $E_{s}$ is the total particle energy and $m_{s}^{\prime}$ is the gyrokinetic magnetic moment. Then, for strongly magnetized AD plasmas a convenient analytical expansion for $\widehat{f_{* s}}$ can be made in terms of the small dimensionless parameter $\varepsilon$. Retaining only the leadingorder expression for the guiding-center magnetic moment $m_{s}^{\prime} \simeq \mu_{s}^{\prime}=\frac{M_{s} w^{\prime 2}}{2 B^{\prime}}$, the following relation holds to first order in $\varepsilon: \widehat{f_{* s}}=\widehat{f}_{s}\left[1+h_{D s}\right]+O\left(\varepsilon^{n}\right), n \geqslant 2$. Here, the zero order distribution $\widehat{f}_{s}$ is expressed as

$$
\widehat{f}_{s}=\frac{n_{s}}{\left(2 \pi / M_{s}\right)^{3 / 2}\left(T_{\| s}\right)^{1 / 2} T_{\perp s}} \exp \left\{-\frac{M_{s}\left(\mathbf{v}-\mathbf{V}_{s}\right)^{2}}{2 T_{\| s}}-\frac{M_{s} w^{\prime 2}}{2 \Delta_{T_{s}}}\right\},
$$

which we will call the $b i$-Maxwellian $K D F$, with $\frac{1}{\Delta_{T_{s}}} \equiv \frac{1}{T_{\perp s}}-\frac{1}{T_{\| s}}$ being related to the temperature anisotropy, while the quantity $h_{D s}$ represents the diamagnetic part of the KDF $\widehat{f_{* s}}$, which depends on the thermodynamic forces associated with the gradients of the fluid fields (Cremaschini et al., (2010)). As a consequence of the asymptotic expansion, fluid moments associated to the stationary KDF can be computed analytically. In particular, the total flow velocity $\mathbf{V}_{s}^{t o t}$ takes on the form $n_{s}^{t o t} \mathbf{V}_{s}^{t o t} \equiv \int d \mathbf{v v} \widehat{f_{* s}} \simeq n_{s}\left[\mathbf{V}_{s}+\Delta \mathbf{U}_{s}\right]$, where by definition $\mathbf{V}_{s}=\Omega_{s}(\psi) R \mathbf{e}_{\varphi}$ is the leading order term, which is purely toroidal, while $\Delta \mathbf{U}_{s}$ represents the self-consistent finite Larmor-radius (FLR) velocity corrections of first order, with both toroidal and poloidal components (Cremaschini, 2010).

\section{The Ampere equation and the "kinetic dynamo"}

In this section we investigate the consequences of the kinetic treatment developed here concerning magnetic field generation, showing that, besides a self-generated poloidal magnetic field, the kinetic equilibrium can also sustain a quasi-stationary toroidal field (kinetic dynamo). This is diamagnetic in origin and is due to the combined effects of FLR corrections and temperature anisotropies. For seeing this, consider the Ampere equation. Using the analytic calculation of the fluid fields discussed in the previous section, this can be written as follows for the self-generated magnetic field:

$$
\nabla \times \mathbf{B}^{\text {self }}=\frac{4 \pi}{c} \sum_{s=i, e} q_{s} n_{s}\left[\mathbf{V}_{s}+\Delta \mathbf{U}_{s}\right]
$$

The toroidal component of this equation gives the generalized Grad-Shafranov equation for the poloidal flux function $\psi_{p}$, which in this approximation becomes:

$$
\frac{\partial b_{\psi_{p}}}{\partial \vartheta}+\frac{\partial b_{\vartheta}}{\partial \psi}=-\frac{4 \pi}{R c} J \sum_{s=e, i} q_{s} n_{s}\left[\Omega_{s}(\psi) R+\Delta_{\varphi s}\right]
$$

where $b_{\psi_{p}} \equiv\left(\frac{J}{R^{2}} \nabla \psi_{p} \cdot \nabla \vartheta\right)$ and $b_{\vartheta} \equiv\left(\frac{J}{R^{2}}\left|\nabla \psi_{p}\right|^{2}\right)$, while $J \equiv \frac{1}{|\nabla \psi \times \nabla \varphi \cdot \nabla \vartheta|}$ is the Jacobian of the coordinate transformations. The remaining terms in Eq.(4.1) give the equation for the toroidal component of the magnetic field $\frac{I(\psi, \vartheta)}{R}$. In the same approximation, this is:

$$
\nabla I(\psi, \vartheta) \times \nabla \varphi=\frac{4 \pi}{c} \sum_{s=i, e} q_{s} n_{s} \frac{\Delta_{3 s}}{B} \nabla \psi \times \nabla \varphi
$$

where $\Delta_{3 s}$ contains the contributions of the species temperature anisotropies. For consistency with the approximation introduced, in the small inverse aspect ratio ordering, it follows that $\frac{\partial I(\psi, \vartheta)}{\partial \vartheta}=0+O\left(\delta^{k}\right)$, i.e., to leading order in $\delta: I=I(\psi)+O\left(\delta^{k}\right)$, with $k \geqslant 1$. This is the only constraint imposed on the kinetic solution by the Ampere equation 
(Cremaschini et al. 2010), which in turn also requires that the corresponding current density in Eq. (4.3) is necessarily a flux function. Then, correct to $O(\varepsilon), O\left(\varepsilon_{M}^{0}\right)$ and $O\left(\delta^{0}\right)$, the differential equation for $I(\psi)$ becomes:

$$
\frac{\partial I(\psi)}{\partial \psi}=\frac{4 \pi}{c} \sum_{s=e, i} q_{s} n_{s} \frac{\Delta_{3 s}}{B},
$$

which uniquely determines an approximate solution for the toroidal magnetic field. This result is remarkable because it shows that there can exist a stationary kinetic dynamo effect which generates an equilibrium toroidal magnetic field without requiring any net accretion and without any possible instability/turbulence phenomena. This new mechanism results from poloidal currents arising due to the FLR effects and temperature anisotropies which are characteristic of the equilibrium KDF for collisionless plasmas. The self-generation of the stationary magnetic field is purely diamagnetic. In particular, the toroidal component is associated with the drifts of the plasma away from the flux surfaces. In the present formulation, possible dissipative phenomena leading to a nonstationary self field have been ignored. Such dissipative phenomena probably do arise in practice and could occur both in the local domain where the equilibrium magnetic surfaces are closed and nested, and elsewhere. Temperature anisotropies are therefore an important physical property of collisionless AD plasmas, giving a possible mechanism for producing a stationary toroidal magnetic field. We stress that this effect disappears altogether in the case of isotropic temperatures.

\section{Conclusions}

In this paper we have presented a kinetic formulation for the quasi-stationary dynamo effect, responsible for the self-generation of magnetic fields in accretion discs around compact objects. The theory, arrived at within the framework of the Vlasov-Maxwell description, is applicable to non-relativistic axi-symmetric collisionless AD plasmas immersed in both gravitational and magnetic fields, the latter being assumed to admit locally closed nested poloidal flux surfaces. In particular, it has been shown that a collisionless AD plasma with temperature anisotropy can produce both poloidal and toroidal asymptotic stationary magnetic fields. For the magnetic field configuration considered here, this may occur even without any net radial accretion flow. This remarkable conclusion can cast further light on the physical mechanisms responsible for the generation of magnetic fields in accretion discs and their structure. Finally, these results can also have important implications for our understanding of the equilibrium properties of accretion discs and their dynamical stability properties.

\section{Acknowledgments}

Work developed in the framework of the Consortium for Magnetofluid Dynamics, Trieste, Italy and the European Research Group on Applied Magnetoscience (GAMAS PDRE), CNRS, France.

\section{References}

Frank J., King A., \& Raine D. 2002, Accretion power in astrophysics, CUP

Cremaschini C., Beklemishev A., Miller J., \& Tessarotto M. 2008, AIP Conf. Proc. 1084, 1067

Cremaschini C., Beklemishev A., Miller J., \& Tessarotto M. 2008, AIP Conf. Proc. 1084, 1073

Szuszkiewicz E. \& Miller J. C. 2001, MNRAS 328, 36

Coppi B. 2005, Phys. Plasmas 12, 057302

Coppi B. \& Rousseau F. 2006, ApJ 641, 458

Cremaschini C., Miller J. C., \& Tessarotto M. 2010, Phys. Plasmas 17, 072902

Catto P. J., Bernstein I. B., \& Tessarotto M. 1987, Phys. Fluids B 30, 2784 